\title{
BUBBLES RISING IN AN INCLINED TWO-DIMENSIONAL TUBE AND JETS FALLING ALONG A WALL
}

\author{
J. LEE ${ }^{\prime}$ and J.-M. VANDEN-BROECK ${ }^{2}$
}

(Received 22 April 1995; revised 23 April 1996)

\begin{abstract}
The motion of a two-dimensional bubble rising at a constant velocity $U$ in an inclined tube of width $H$ is considered. The bubble extends downwards without limit, and is bounded on the right by a wall of the tube, and on the left by a free surface. The same flow configuration describes also a jet emerging from a nozzle and falling down along an inclined wall. The acceleration of gravity $g$ and the surface tension $T$ are included in the free surface condition. The problem is characterized by the Froude number $F=U / \sqrt{g H}$, the angle $\beta$ between the left wall and the horizontal, and the angle $\gamma$ between the free surface and the right wall at the separation point. Numerical solutions are obtained via series truncation for all values of $0<\beta<\pi$. The results extend previous calculations of Vanden-Broeck [12-14] for $\beta=\pi / 2$ and of Couët and Strumolo [3] for $0<\beta<\pi / 2$. It is found that the behavior of the solutions depends on whether $0<\beta<2 \pi / 3$ or $2 \pi / 3 \leq \beta<\pi$. When $T=0$, it is shown that there is a critical value $F_{c}$ of Froude number for each $0<\beta<2 \pi / 3$ such that solutions with $\gamma=0, \pi / 3$ and $\pi-\beta$ occur for $F>F_{c}, F=F_{c}$ and $F<F_{c}$ respectively, and that all solutions are characterized by $\gamma=0$ for $2 \pi / 3 \leq \beta<\pi$. When a small amount of surface tension $T$ is included in the free surface condition, it is found that for each $0<\beta<\pi$ there exists an infinite discrete set of values of $F$ for which $\gamma=\pi-\beta$. A particular value $F^{*}$ of the Froude number for which $T=0$ and $\gamma=\pi-\beta$ is selected by taking the limit as $T$ approaches zero. The numerical values of $F^{*}$ and the corresponding free surface profiles are found to be in good agreement with experimental data for bubbles rising in an inclined tube when $0<\beta<\pi / 2$.
\end{abstract}

\section{Introduction}

We consider a bubble rising at a constant speed $U$ in an inclined two-dimensional tube of width $H$ (see Figure 1a). The bubble is bounded on the right by a wall of the tube and on the left by a free surface. We assume that the tube is infinitely long and that

\footnotetext{
'Department of Mathematics, Kwang Woon University, Wolgye-dong Nowon-Gu, Seoul 139-701, Korea ${ }^{2}$ Department of Mathematics and Center for the Mathematical Sciences University of Wisconsin Madison, Madison, WI 53706

(C) Australian Mathematical Society, 1998, Serial-fee code 0334-2700/98
} 


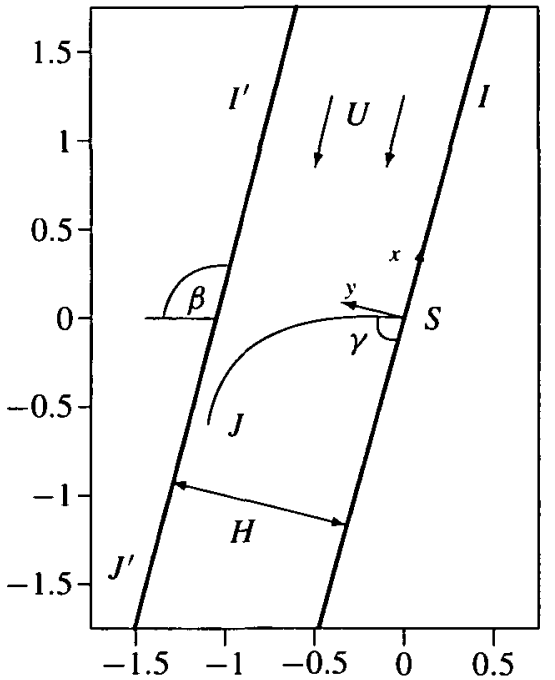

(a)

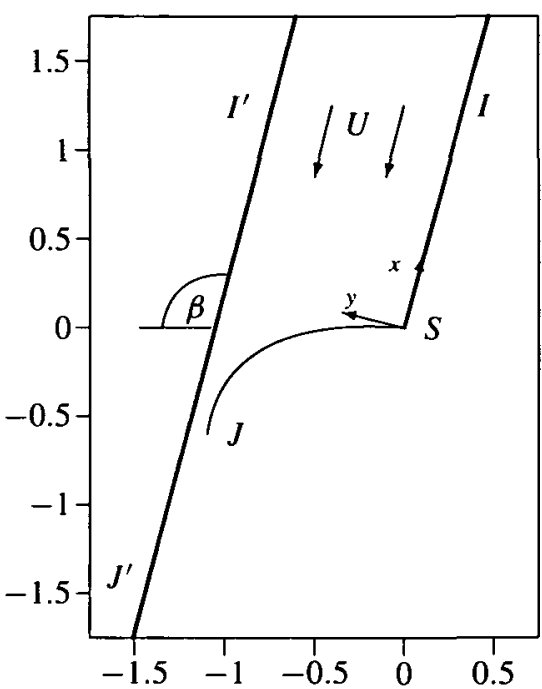

(b)

FIGURE 1. Sketch of the flow domain and the coordinates. This is a computed profile for $\beta=7 \pi / 12$ and $F=0.11$ and $\omega=10$.

the bubble extends downwards without limit. We choose a frame of reference moving with the bubble. Gravity is acting vertically downwards. The effect of the surface tension $T$ is included in the dynamic boundary condition. The angle between the left wall and the horizontal is denoted by $\beta$ and the angle between the negative $x$-axis and the tangent line at the nose of the bubble by $\gamma$ (see Figure 1a). The flow configuration of Figure 1a describes also a jet emerging from a nozzle and falling down along a wall. In this case the flow is viewed as bounded on the left by an infinite wall and on the right by a semi-infinite wall and a free surface (see Figure $1 \mathrm{~b}$ ).

The bubble of Figure 1a has been studied experimentally by Maneri [9] for $0<\beta<\pi / 2$. No experimental results have been reported for $\pi / 2<\beta<\pi$, because the bubble tends to rise on the upper wall. Various configurations of jets falling from a nozzle or an aperture have been considered analytically by Keller and Gee [7] and numerically by Tuck [11], Dias and Vanden-Broeck [4], Lee and VandenBroeck [8] and others. These authors included gravity but neglected surface tension. Our calculations generalize some of their findings by including surface tension. We consider solutions for the flow of Figure $1 \mathrm{~b}$ for all values of $0<\beta<\pi$, although the solutions for $\pi / 2<\beta<\pi$ are "upside down flows" with the heavy fluid on top of the light fluid. We have two motivations for considering such flows. First, "upside down flows" are commonly observed. For example, when a liquid is poured from a container, it sometimes flows along the underside of the spout of the pouring vessel. This phenomenon was studied experimentally by Reiner [10], who called it 
the "teapot effect". Analytical and numerical solutions of such pouring flows have been found by Keller [6] and Vanden-Broeck and Keller [15, 16]. Secondly, it is of interest to describe the local behavior of the flow near the separation point (that is, the point of intersection of the free surface with the right wall) for all $0<\beta<\pi$, and in particular, the dependence of $\gamma$ on $\beta$.

For each value of $\beta$, the problem is characterized by the Froude number

$$
F=U / \sqrt{g H}
$$

and the Weber number

$$
\omega=\rho U^{2} H / T
$$

Here, $\rho$ is the density of the fluid.

Garabedian [5], Birkhoff and Carter [1] and Vanden-Broeck [12-14] considered the problem of a free bubble rising in a two-dimensional tube. This configuration can be obtained by reflecting the flow of Figure la with $\beta=\pi / 2$ into the right wall. After reflection the width of the tube is $2 H$ (see Figure 2). As we shall use results from Vanden-Broeck [12-14] later, we summarize them here as follows. When $\omega=\infty$ (that is, $T=0$ ), there is a critical value $F_{c} \approx 0.506$ of the Froude number $F$ such that

$$
\gamma=\pi / 2 \text { for } F<F_{c}, \quad \gamma=\pi / 3 \text { for } F=F_{c}, \quad \gamma=0 \text { for } F>F_{c} .
$$

We note that Vanden-Broeck ([12-14]) used a Froude number (which we denote here by $F_{r}$ ) based on the width of the tube. Thus $F_{r}$ is related to $F$ by

$$
F_{r}=F / \sqrt{2} \text {. }
$$

The mathematical solution for $\beta=\pi / 2$ has three interpretations: a jet falling from a nozzle (see Figure lb), a bubble bounded on the right by a wall and on the left by a free surface (see Figure 1a) and a free bubble (see Figure 2).

If we use the interpretation of Figure 1b, relations (1.3) agree with our everyday experience: there is a jet for each value of $F$.

If we use the interpretation of Figure 2, we need to require $\gamma=\pi / 2$ so that the slope is continuous at the nose of the bubble. Relations (1.3) show that there is a solution for each value of $0<F<F_{c}$. This is in contradiction with the experiments of Collins [2] which show that there is only one bubble corresponding to $F \approx 0.35$. Vanden-Broeck [13] showed that the discrepancy between the experiments and the theory can be removed by introducing surface tension and taking the limit as the surface tension approaches zero. Vanden-Broeck [13] showed that when $\omega \neq \infty$ (that is, $T \neq 0$ ), the angle $\gamma$ is a function of $\omega$ and $F$. For a fixed value of $\omega, \gamma$ oscillates 


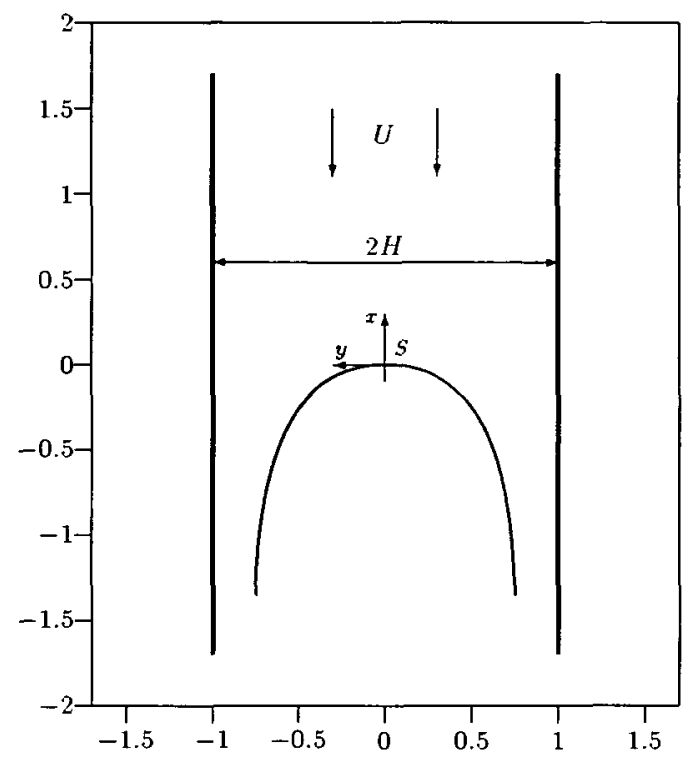

FIGURE 2. Computed solutions for $\beta=\pi / 2$ and $F=F^{*} \approx 0.325$.

infinitely often around $\pi / 2$ as $F$ decreases. There is a countably infinite number of values of $F$ for which $\gamma=\pi / 2$. Furthermore, as $\omega \rightarrow \infty$, all the solutions approach a unique solution characterized by $F^{*} \approx 0.325$. Therefore an arbitrary small amount of the surface tension can be used to select a particular solution with $T=0$ and $\gamma=\pi / 2$ (in the absence of surface tension, all solutions for $0<F<F_{c}$ are characterized by $\gamma=\pi / 2$ ). The value $F^{*} \approx 0.325$ is in good agreement with the experimental value 0.35 of Collins [2].

The flow problem with the interpretation of Figure la was solved numerically by Couët and Strumolo [3]. They restrict their attention to $0<\beta<\pi / 2$. Their results showed that for given values of $\beta$ and $\omega, \gamma$ oscillates infinitely often as $F$ approaches zero. For each value of $0<\beta<\pi / 2$ and $\omega$, they selected the particular solution corresponding to the largest value of $F$ for which $\gamma=\pi / 2$.

In this paper we extend the calculations of Vanden-Broeck [12-14] and Couët and Strumolo [3] and present numerical solutions of the flow configurations of Figures la and $\mathrm{lb}$ for values of $0<\beta<\pi$.

The numerical procedure uses series truncation. It is similar to the techniques used by Vanden-Broeck [12-14], Couët and Strumolo [3] and Lee and Vanden-Broeck [8]. It is found that the behaviors of the solutions depend on whether $0<\beta<2 \pi / 3$ or $2 \pi / 3 \leq \beta<\pi$. 
For $T=0$ and each $0<\beta<2 \pi / 3$, there is a critical value $F_{c}$ of the Froude number $F$, such that solutions with $\gamma=0, \pi / 3$ and $\pi-\beta$ occur for $F>F_{c}, F=F_{c}$ and $F<F_{c}$, respectively. This includes (1.3) as a particular case for $\beta=\pi / 2$. On the other hand, for $T=0$ and each value of $2 \pi / 3 \leq \beta<\pi$, there is no such a critical value of $F$ and $\gamma=0$ for all $0<F<\infty$.

When the flow is interpreted as in Figure 1a, surface tension can be used to select the physically relevant bubble. We propose a new selection criterion and show that the selected values of the Froude number and the selected profiles are in good agreement with the experiments of Maneri [9].

The problem is formulated in Section 2. The numerical procedure is described in Section 3 and the results are presented and discussed in Sections 4, 5 and 6.

\section{Formulation}

Let us consider the steady two-dimensional potential flow of an inviscid incompressible fluid in a domain bounded on the left by an infinite wall $I^{\prime} J^{\prime}$ and on the right by a semi-infinite wall $I S$ and a free surface $S J$ (see Figure la). As $x \rightarrow \infty$, the velocity approaches the constant $U$. We introduce Cartesian coordinates with the origin at the separation point $S$. Gravity acts vertically downwards. Then, the gravitational potential is given by

$$
G=g x \sin \beta-g y \cos \beta .
$$

On the free surface $S J$, the Bernoulli's equation yields

$$
\frac{1}{2} q^{2}+G-(T / \rho) K=B,
$$

where $q$ is the flow speed, $K$ is the curvature of the free surface and $B$ is the Bernoulli constant.

We define dimensionless variables by taking $U$ as the unit velocity and $H$ as the unit length. We denote the potential function by $\phi$ and the stream function by $\psi$. In addition, we introduce the complex velocity by $\zeta=u-i v$, and we define the function $\tau-i \theta$ by the relation

$$
\zeta=u-i v=e^{\tau-i \theta} .
$$

We denote the complex potential by $f=\phi+i \psi$. Without loss of generality, we choose $\phi=\psi=0$ at $x=y=0$. It follows from the choice of the dimensionless variables that $\psi=0$ on the surface of the bubble $S J$ and $\psi=-1$ on the wall $I^{\prime} J^{\prime}$. The complex potential plane is sketched in Figure 3. We shall seek the function $\tau-i \theta$ as an analytic function of $f$ in the strip $-1<\psi<0$. 


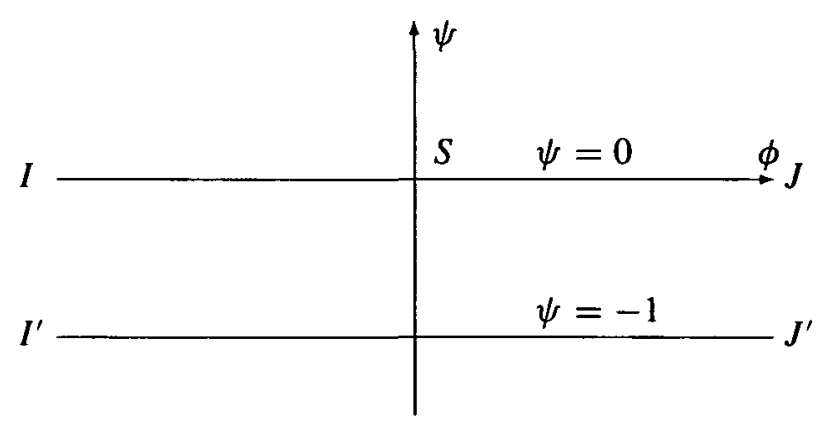

FIGURE 3. The complex potential plane.

In terms of the dimensionless variables, (2.2) becomes

$$
e^{2 \tau}+\frac{2}{F^{2}}(x \sin \beta-y \cos \beta)-\frac{2}{\omega} e^{\tau}\left|\frac{\partial \theta}{\partial \phi}\right|=\frac{B}{U^{2}} .
$$

Here, $F$ and $\omega$ are the Froude number and the Weber number defined by (1.1) and (1.2), respectively.

Differentiating (2.4) with respect to $\phi$ and using the relation

$$
\frac{\partial x}{\partial \phi}+i \frac{\partial y}{\partial \phi}=\frac{1}{u-i v}=e^{-\tau+i \theta},
$$

we have

$$
e^{2 \tau} \frac{\partial \tau}{\partial \phi}+\frac{1}{F^{2}} e^{-\tau} \sin (\beta-\theta)-\frac{1}{\omega} \frac{\partial}{\partial \phi}\left(e^{\tau} \frac{\partial \theta}{\partial \phi}\right)=0 \text { on } S J
$$

The kinematic conditions on the walls $I^{\prime} J^{\prime}$ and $I S$ yield

$$
\begin{gathered}
\theta=\pi, \psi=-1, \\
\theta=\pi, \psi=0, \phi<0 .
\end{gathered}
$$

This completes the formulation of the problem of determining $\tau-i \theta$. This function must be analytic in the strip $-1<\psi<0$ and satisfy the conditions (2.6)-(2.8).

\section{Numerical Procedures}

We solve the problem by following the procedures used by Vanden-Broeck [12-14], Couët and Strumolo [3] and Lee and Vanden-.Broeck [8]. 


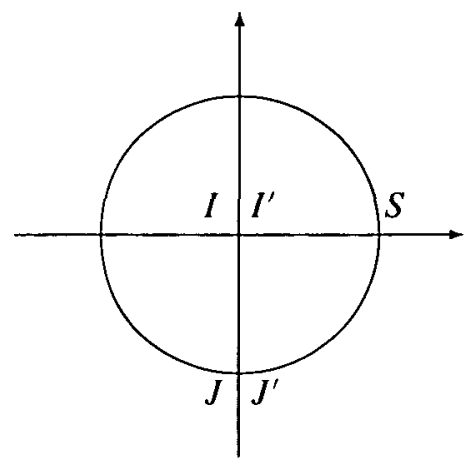

FIGURE 4. The complex $t$-plane.

We first map the flow domain on the fourth quadrant of the unit circle in the complex $t$-plane by the transformation

$$
e^{-\frac{\pi}{2} f}=\frac{1}{2}\left(t+\frac{1}{t}\right)
$$

The wall $I S$ goes onto the real interval $(0,1)$ and the bubble surface $S J$ onto the circumference (see Figure 4).

Next we define the function $\Omega(t)$ by the relation

$$
\zeta=e^{\tau-i \theta}=-\left[-\ln C\left(1+t^{2}\right)\right]^{1 / 3}(-\ln C)^{-1 / 3}\left(1-t^{2}\right)^{2 \gamma / \pi} e^{\Omega(t)}
$$

Here $C$ is an arbitrary constant between 0 and 0.5 . We choose $C=0.2$. At the points $J$ and $S, \zeta$ has singularities associated with a thin jet and a flow inside an angle of $\pi-\gamma$ (see Figure 1a). These singularities are removed in (3.2) by the factors $\left[-\ln C\left(1+t^{2}\right)\right]^{1 / 3}$ and $\left(1-t^{2}\right)^{2 \gamma / \pi}$ (see Birkhoff and Carter [1], Vanden-Broeck [1214] and Lee and Vanden-Broeck [8] for details). It follows that $\Omega(t)$ can be represented by a Taylor expansion in powers of $t$. Furthermore, the kinematic conditions (2.7) and (2.8) imply that the expansion for $\Omega(t)$ has real coefficients $a_{n}$ and involves only even powers of $t$. Thus we write

$$
\Omega(t)=\sum_{n=1}^{\infty} a_{n} t^{2 n}
$$

We describe points on the free surface $S J$ by $t=e^{i \sigma}$, where $-\pi / 2<\sigma \leq 0$. Using (3.1), we rewrite (2.6) in the form

$$
\pi \cot \sigma e^{2 \tilde{\tau}} \frac{d \tilde{\tau}}{d \sigma}+\frac{2}{F^{2}} e^{-\tilde{\tau}} \sin (\beta-\tilde{\theta})-\frac{\pi^{2}}{2 \omega} \cot \sigma \frac{d}{d \sigma}\left(e^{\tilde{\tau}} \cot \sigma \frac{d \tilde{\theta}}{d \sigma}\right)=0 .
$$


Here, $\tilde{\tau}(\sigma)$ and $\tilde{\theta}(\sigma)$ denote the values of $\tau$ and $\theta$ on the free surface $S J$.

We solve the problem by truncating the infinite series in (3.3) after a finite number of terms.

We first consider the case $\omega=\infty$ (that is, $T=0$ ). The local analysis of Lee and Vanden-Broeck [8] shows that the only possible values of $\gamma$ are $0, \pi / 3$ and $\pi-\beta$. Therefore, we have three different schemes corresponding to each of these values of $\gamma$. In each of them, we truncate the infinite series in (3.3) after $N$ terms and satisfy the free surface condition (3.4) at the $N$ mesh points

$$
\sigma_{I}=\frac{\pi}{2 N}\left(I-\frac{1}{2}\right), \quad I=1,2, \ldots, N .
$$

This leads a system of $N$ nonlinear algebraic equations for the $N$ unknowns $a_{n}$, $n=1,2, \ldots, N$. We solve this system by Newton's method. Once this system is solved for given values of $F$ and $\omega$ (for each $\beta$ ), the shape of the bubble is obtained by numerically integrating $(2.5)$.

We now consider the case $\omega \neq \infty$ (that is, $T \neq 0$ ). The angle $\gamma$ is no longer restricted to the values $0, \pi / 3$ and $\pi-\beta$ and must be found as part of the solution. Thus we truncate the infinite series in (3.3) after $N-1$ terms and satisfy (3.4) at the $N$ collocation points (3.5). This leads to a system of $N$ nonlinear algebraic equations for the $N$ unknowns $a_{n}, n=1,2, \ldots, N-1$ and $\gamma$. This system is also solved by Newton's method.

\section{Numerical results}

The numerical schemes of Section 3 were used to compute solutions for various values of $F, \omega$ and $\beta$. We found that the coefficients $a_{n}$ decrease rapidly as $n$ increases. For example, $a_{1} \sim 1.6 \times 10^{-1}, a_{10} \sim 5.7 \times 10^{-3}, a_{30} \sim 2.2 \times 10^{-3}$ and $a_{50} \sim 2.5 \times 10^{-4}$ for $\beta=7 \pi / 12, \omega=10$ and $F=0.14$ when $N=70$. Most of the computations were performed with $70 \leq N \leq 200$. We also repeated the calculations with $C \neq 0.2$ and checked that the results were independent of the value of $0<C<0.5$.

I. Solutions without surface tension. When surface tension is neglected, there is a solution for each value of $F$ and $\beta$. As explained in Section 3, the angle $\gamma$ takes only the values $0, \pi / 3$ and $\pi-\beta$. The numerical results show that there is a critical Froude number $F_{c}$ for each $0<\beta<2 \pi / 3$ such that

$$
\gamma=\pi-\beta \text { when } F<F_{c}, \quad \gamma=\pi / 3 \text { when } F=F_{c}, \quad \gamma=0 \text { when } F>F_{c},
$$

and that for $2 \pi / 3 \leq \beta<\pi$ there is only one configuration with $\gamma=0$ for each $F>0$. 


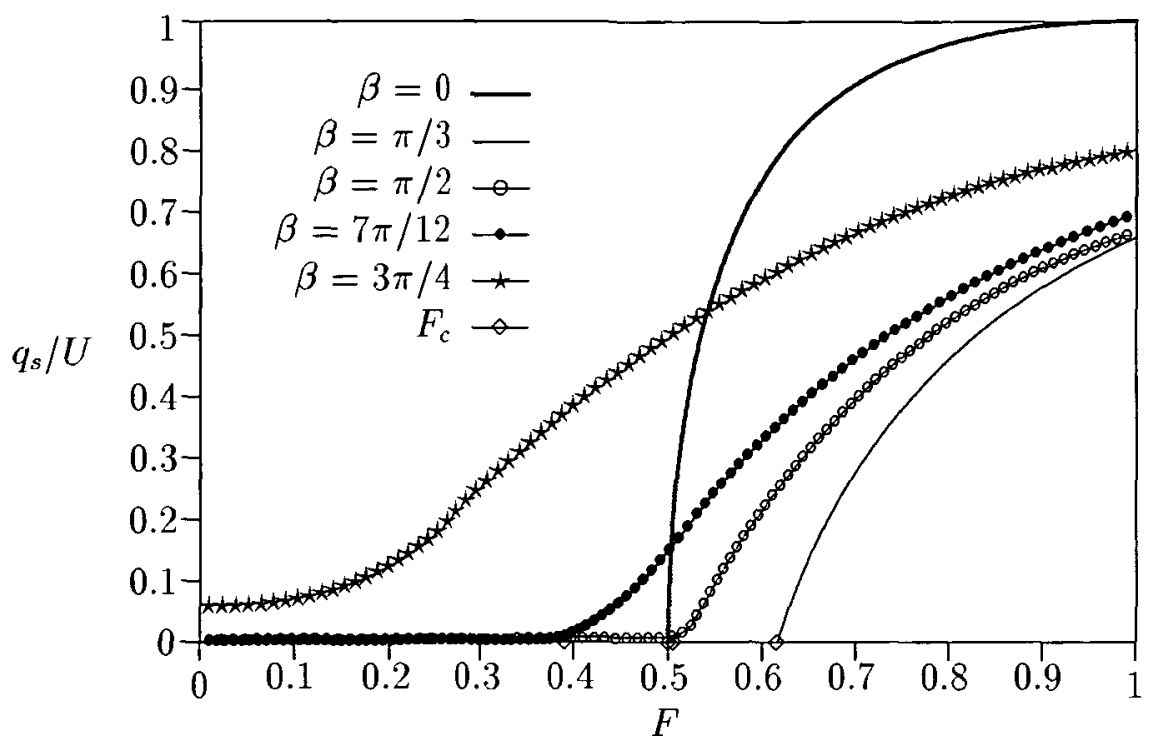

FIGURE 5. Values of the speed $q_{s} / U$ versus $F$ for various values of $\beta$.

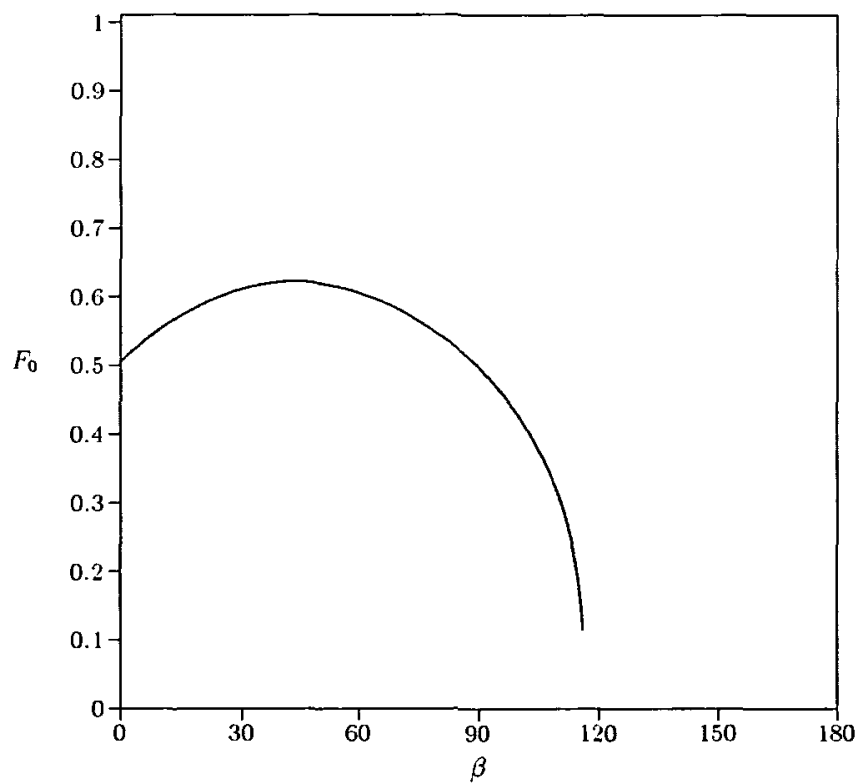

FIGURE 6. Values of $F_{c}$ versus $\beta$.

We denote by $q_{S}$ the velocity at the separation point $S$. The solutions with $\gamma=0$ 
are also characterized by $q_{s} \neq 0$, and those with $\gamma=\pi / 3$ and $\gamma=\pi-\beta$ by $q_{s}=0$. For $2 \pi / 3 \leq \beta<\pi, q_{s}$ tends to zero as $F$ approaches zero. Figure 5 shows the values of $q_{s}$ versus $F$ for various $\beta$. The results of Figure 5, restricted to $0<\beta<\pi / 2$, are similar to those in Figure 11 of Couët and Strumolo [3]. Note that the inclination $\beta$ used by Couët and Strumolo (which we denote by $\beta_{c}$ ) is the complement of our angle $\beta$. Thus $\beta_{c}=\pi / 2-\beta$.

Figure 6 shows the values of $F_{c}$ versus $\beta$. For $\beta=\pi / 2, F_{c} \approx 0.506$, in accordance with Vanden-Broeck's [12] results. As $\beta$ approaches $2 \pi / 3$ from below, larger and larger values of $N$ are needed to obtain accurate values of $F_{c}$. This numerical difficulty is related to the fact that there are no solutions with $\gamma=\pi / 3$ for $\beta>2 \pi / 3$. However an extrapolation of the results of Figure 6 confirms that $F_{c}$ approaches zero as $\beta$ tends to $2 \pi / 3$ from below.

In Figures 7a, 7b and 7c, we present typical profiles for several values of $\beta$. As $F \rightarrow \infty$, the profile of the bubble approaches the wall. As $F \rightarrow 0$, the profile of the bubble approaches a horizontal line. When $0<\beta<2 \pi / 3$, the bubble surface at the contact point $S$ is tangent to the wall for each value of $F_{c}<F<\infty$ and leaves horizontally from the wall for each $0<F<F_{c}$ (see Figure 7a and 7b). On the other hand, for $2 \pi / 3 \leq \beta<\pi$, the free surface leaves tangentially from the point $S$ for all $F>0$ ( see Figure 7 c).

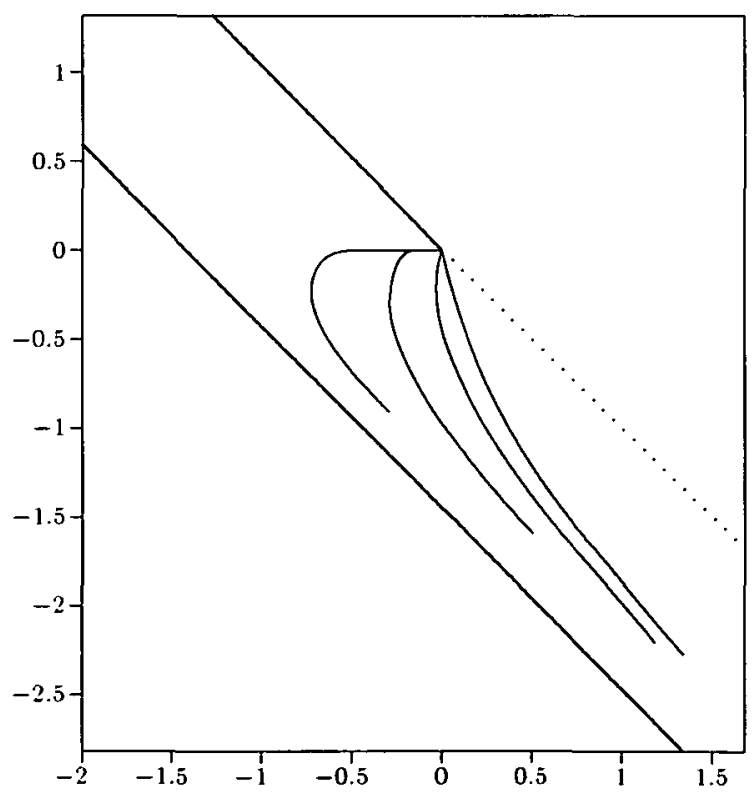

FIGURE 7A. Computed solutions for $\beta=\pi / 4$ and $T=0$. The free surface profiles from left to right correspond to $F=0.2,0.45, F=F_{c} \approx 0.6262$ and $F=0.8(q, \approx 0.491)$. 


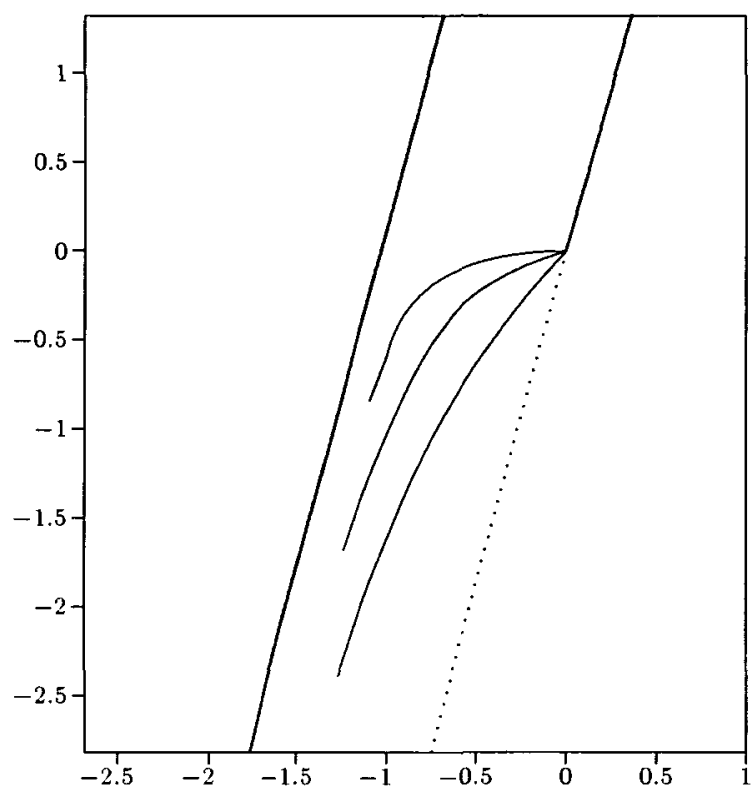

FIGURE 7B. Computed solutions for $\beta=7 \pi / 12$ and $T=0$. The free surface profiles from left to right correspond to $F=0.18, F=F_{c} \approx 0.393$ and $F=0.8$.

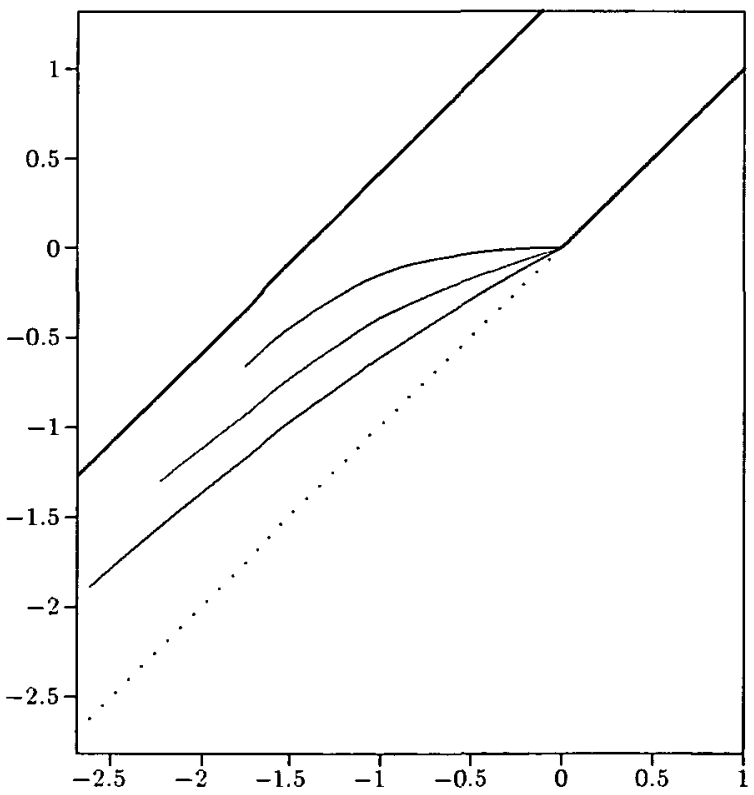

FIGURE 7C. Computed solutions for $\beta=3 \pi / 4$ and $T=0$. The free surface profiles from left to right correspond to $F=0.2\left(q_{s} \approx 0.121\right), F=0.5\left(q_{s} \approx 0.503\right)$ and $F=0.9\left(q_{s} \approx 0.761\right)$. 
II. Solutions with surface tension. When surface tension is included in the free surface condition, the numerical computations show that there is a flow for each value of $F, \beta$ and $\omega$. As mentioned in Section 3, the angle $\gamma$ comes as part of the solution and its values are no longer restricted to $0, \pi / 3$ and $\pi-\beta$. In Figure 8 , we present values of $\gamma$ versus $F$ for $\omega=10$ and several values of $0<\beta<\pi$. These results confirm the calculations of Couët and Strumolo [3] for $0<\beta<\pi / 2$ and extend them to the range $0<\beta<\pi$ (see their Figure 6). For each value of $\beta$ we found that $\gamma$ tends to zero as $F$ tends to infinity. Figure 8 shows that for a fixed value of $\omega$, the amplitude of the oscillation around $\gamma=\pi-\beta$ on each curve dies out as $\beta$ increases. Similar curves were obtained for other values of $\omega$ sufficiently large. We observed that the amplitude of the oscillations decreases as $\omega$ increases when $\beta$ is fixed.

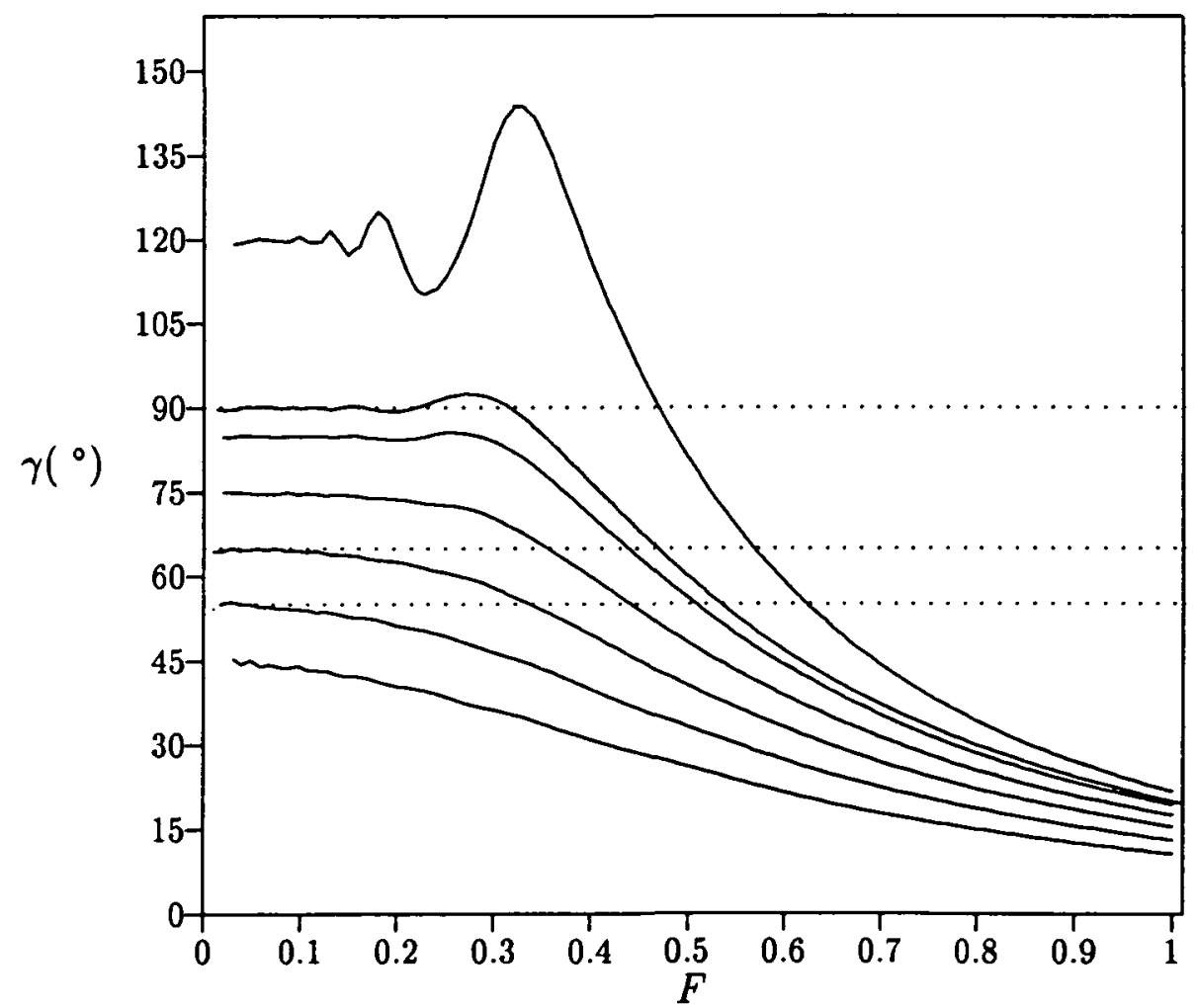

FIGURE 8. Values of $\gamma$ versus $F$ for $\omega=10$. The curves from the top to the bottom correspond to $\beta=\pi / 3, \pi / 2,19 \pi / 36,7 \pi / 12,23 \pi / 36,25 \pi / 36$ and $3 \pi / 4$. The dotted lines from the top to bottom correspond to the value $\gamma=\pi-\beta$ for $\beta=\pi / 2,23 \pi / 36$ and $25 \pi / 36$, respectively.

Typical profiles for $\beta=3 \pi / 4$ with $\omega=1$ are shown in Figure 9. We note that equation (2.4) implies that the free surface approaches a horizontal line for any $\omega$ and $\beta$ as $F$ tends to zero. 


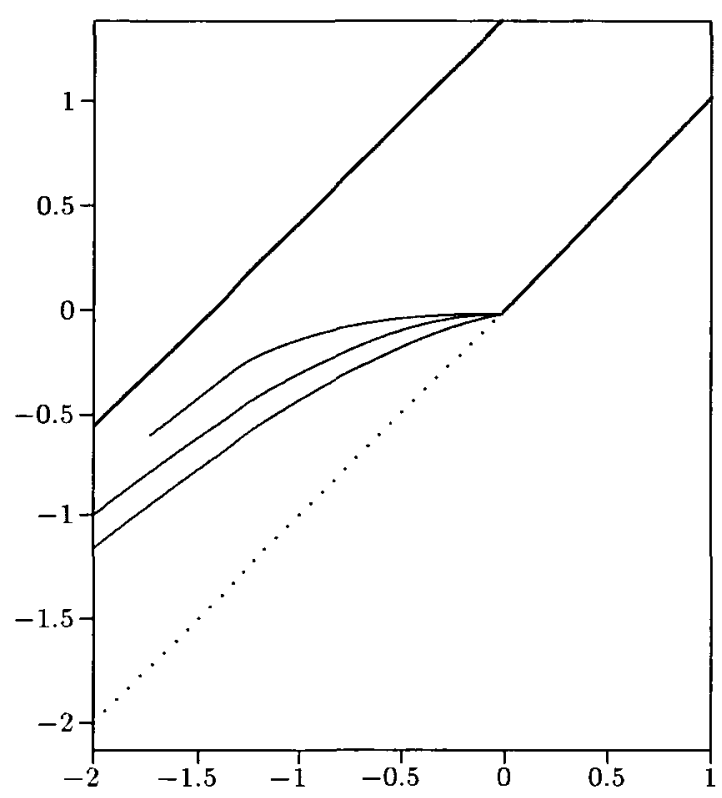

FIGURE 9. Computed solutions for $\beta=3 \pi / 4$ and $\omega=1$. The curves from left to right correspond to $F=0.2,0.4$ and 0.6 .

\section{Discussion of the results, selection technique and comparison with experiments}

As we mentioned in the introduction, the flow configuration studied in this paper describes two different physical problems.

The first is a jet falling down along a wall (see Figure $l b$ ). In this case, the numerical results with $T=0$ show that there is a jet for each value of $F$ and $0<\beta<\pi$.

The second physical problem is a bubble rising in an inclined tube (see Figure 1a). The numerical results with $T=0$ show that there is a mathematical solution for each value of $0<\beta<\pi$ and $F$. Recall that $\beta$ is defined as the angle between the left wall and the horizontal (see Figure 1a). For any value of $0<\beta_{1}<\pi / 2$, the angles $\beta=\beta_{1}$ and $\beta=\pi-\beta_{1}$ correspond to the same inclined tube viewed from the front or the back. Therefore there are two solutions for a given inclined tube. However, solutions with $\pi / 2<\beta<\pi$ have not been observed experimentally. Also, the experimental data (Maneri [9]) show that for each $0<\beta<\pi / 2$, there is only one value of $F$ for which a bubble exists. This does not agree with the numerical results which predict a solution for each values of $F$ and $0<\beta<\pi / 2$. The discrepancy can removed by generalizing the procedure derived by Vanden-Broeck [13] for the configuration of Figure 2 (see the introduction for a summary of the method). Thus we introduce 
surface tension and take the limit as the surface tension approaches zero. The details are described below.

Couët and Strumolo [3] choose for each value of $\beta$ and $\omega$, the particular solution corresponding to the largest value of $F$ for which $\gamma=\pi / 2$. Our results show that there are no solutions with $\gamma=\pi / 2$ when $T=0$ unless $\beta=\pi / 2$ (see (4.1)). This finding does not invalidate the selection criterion of Couët and Strumolo because they use it with $T$ small but different from zero. In fact their selected solutions are in very good agreement with experiments as shown for example in the Figures 15, 16 and 17 of their paper.

Here we show that an equally good agreement with experiments can be obtained by a different selection criterion in which we take the limit as $T \rightarrow 0$ instead of keeping $T \neq 0$ as in Couët and Strumolo [3]. More precisely we select for each $0<\beta<\pi / 2$ the value $F^{*}$ of the Froude number defined by

$$
F^{*}=\lim _{\omega \rightarrow \infty} F_{\beta}(\omega)
$$

where $F_{\beta}(\omega)$ is the largest Froude number at which $\gamma=\gamma_{0}$, for given values of $0<\beta<\pi / 2$ and $\omega$. The results of Section 4I show that $\gamma_{0}$ can only take one of the values $\pi-\beta, \pi / 3$ and 0 (that is, one of the only three possible values in the absence of surface tension).

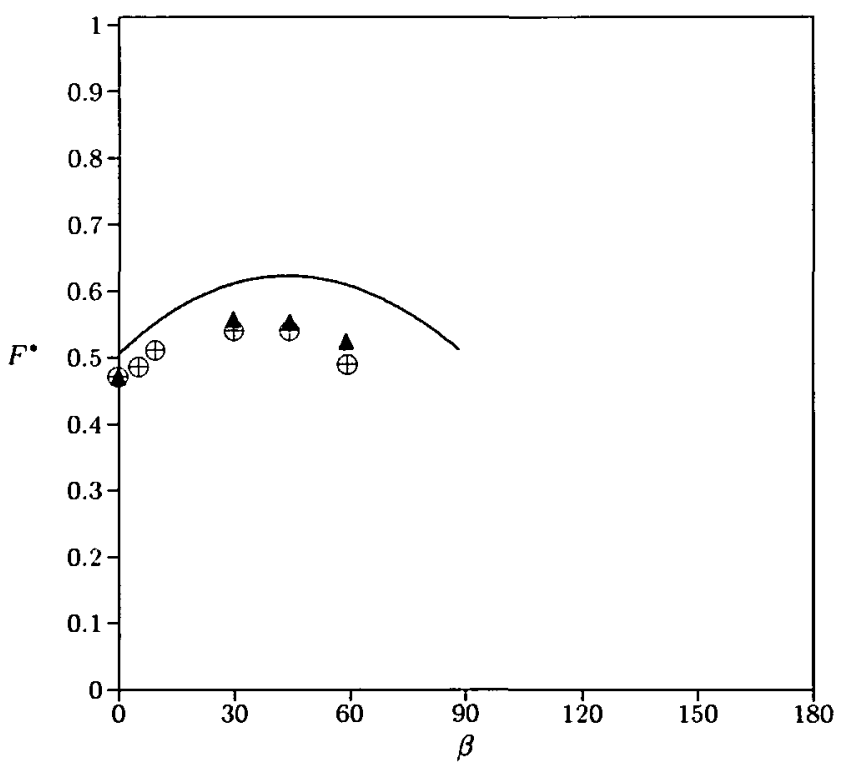

FIGURE 10. Values of $F_{\circ}$ versus $\beta$. The dots are the experimental values of Maneri (1970); $\boldsymbol{\Lambda}$, methanol; $\oplus$, water. 
Figure 8 shows that $F_{\beta}(\omega)=\infty$ for $\gamma=0$ and $\omega$ sufficiently large. Thus our criterion (4.2) with $\gamma_{0}=0$ leads to the uninteresting value $F^{*}=\infty$. The only solutions with $\gamma=\pi / 3$ in the absence of surface tension corresponds to $F=F_{c}$ (see (4.1)). Therefore our criterion with $\gamma_{0}=\pi / 3$ leads to $F^{*}=F_{c}$. In Figure 10, we compare these selected values with the experimental values of Maneri [9]. The agreement is not very satisfactory and the selected values are too high.

For each value of $\omega \neq \infty$ and $0<\beta<\pi / 2$, Figure 8 shows that there is a discrete set of values for which $\gamma=\pi-\beta$. The values $F^{*}$ selected by our criterion for $\gamma=\pi-\beta$ are shown in Figure 11. In order to find these values for each $0<\beta<\pi / 2$, we computed first $F_{\beta}(\omega)$ by using a variant of the scheme of Section 3 in which the problem is reduced to a system of $N$ algebraic equations for $N$ unknowns $F$ and $a_{n}, n=1,2, \ldots, N-1$ to be solved for $\gamma=\pi-\beta$ and given values of $\beta$ and $\omega$. We then determined the value of $F^{*}$ for each $\beta$ by evaluating $F_{\beta}(\omega)$ for larger and larger values of $\omega$.

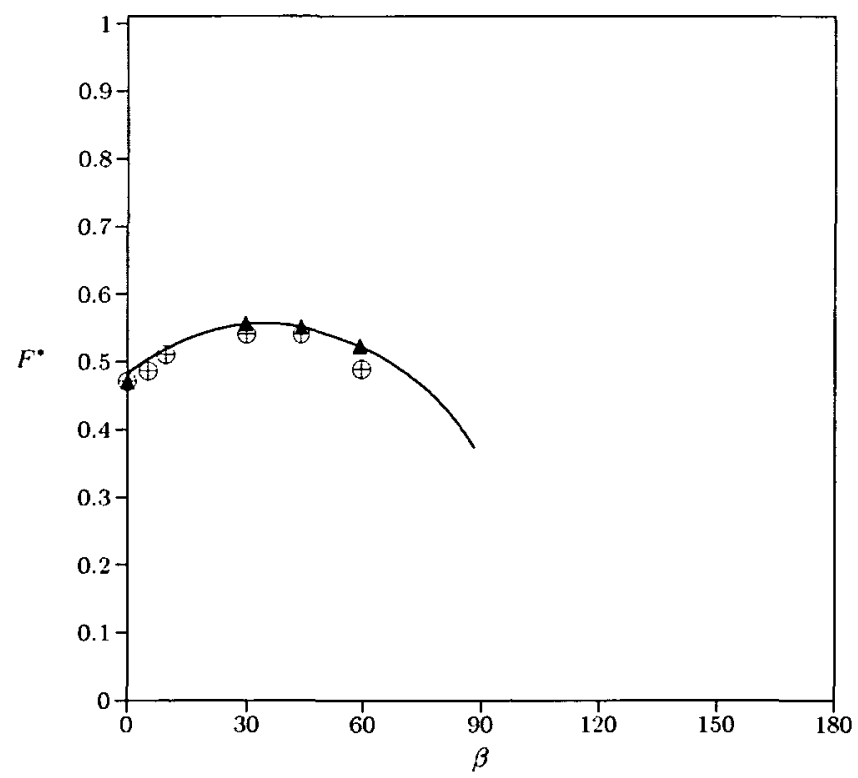

FIGURE 11. Values of $F^{*}$ versus $\beta$. The dots are the experimental values of Maneri (1970); $\Delta$, methanol; $\oplus$, water.

We also show in Figure 11 the experimental values of Maneri [9]. The agreement between experiments and the theoretical curve is uniformly good. This finding indicates that our criterion should be used with $\gamma_{0}=\pi-\beta$. The agreement is better for methanol than water. This is to be expected since the value of the surface tension $T$ for methanol is 3 times smaller than for water (recall that our selection criterion 
applies in the limit as $T$ approaches zero).

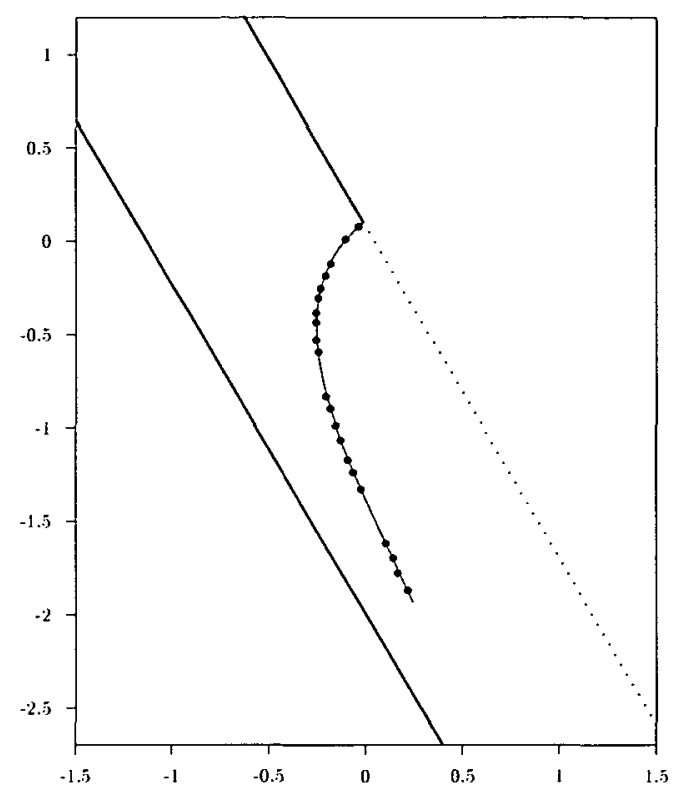

FIGURE 12. Computed solution for $\beta=\pi / 3$ and $F=F^{*} \approx 0.527$. The dots are the experimental values of Maneri (1970).

A free surface profile for $\beta=\pi / 3$ and $F=F^{*} \approx 0.527$ is shown in Figure 12 .

We also compared in Figure 12 our selected profile for $\beta=\pi / 6$ with the same experimental values as Couët and Strumolo in their Figure 15. The agreement is also very good and supports our choice $\gamma_{0}=\pi-\beta$ in (4.2).

It is worthwhile mentioning that our selection criterion can be generalized by replacing $F_{\beta}(\omega)$ in (4.2), by the $n-t h$ largest Froude number at which $\gamma=\pi-\beta$ for given values of $0<\beta<\pi / 2$ and $\omega$. Here $n$ denotes an integer. Since both the amplitude and the wavelength of the oscillations around $\pi-\beta$ in Figure 8 tend to zero as $T \rightarrow 0$, the criterion yields the same value of $F^{*}$ independently of the value of $n$.

Finally we consider further the case $\beta=\pi / 2$. As described in the introduction there are two different possible bubbles. The first one is a bubble bounded on the left by a free surface and on the right by a wall of the tube (see Figure 1a with $\beta=\pi / 2$ ). The corresponding selected value of $F^{*}$ is 0.325 . The second one is the free bubble of Figure 2. The experiments of Collins [2] and Maneri [9] show that the second bubble is the one which is observed. The selected value of the Froude number based on the width of the tube for the free bubble of Figure 2 was found by Vanden-Broeck [13] to be $F_{r}=0.23$ (see introduction again for details). This values of 0.23 does not 
agree with the value 0.325 predicted in Figure 11 for $\beta=\pi / 2$. The reason for this discrepancy is that the free bubble solution of Figure 2 is part of another family of solutions which is not described by our model, namely a free bubble in an inclined tube (see Figure 13 for a sketch). It is only in the case $\beta=\pi / 2$ that the free bubble can be obtained from the configuration of Figure $1 \mathrm{a}$ by a simple reflection. The experiments of Maneri [9] suggest that the branch of free bubbles exist only for values of $\beta$ close to $\pi / 2$. As $\beta$ decreases from $\pi / 2$, there is a transition to the solutions described in this paper.

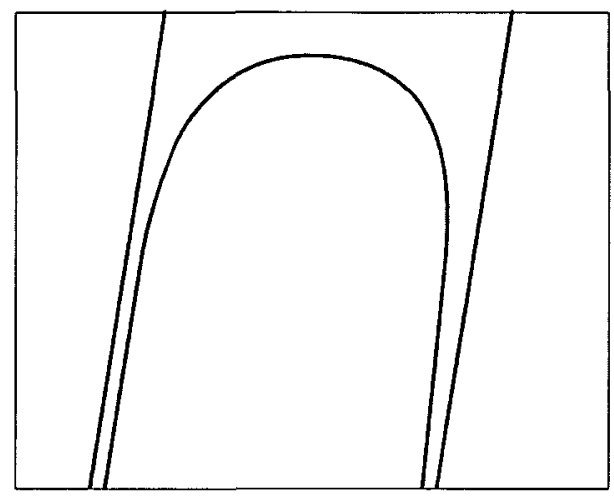

FIGURE 13. Sketch of a free bubble rising in an inclined tube.

\section{Conclusions}

We have presented numerical solutions for a flow bounded by two walls and a free surface. The configuration models a bubble rising in a tube or a jet falling down from a nozzle along a wall. When surface tension is neglected there are solutions corresponding to three different values of the angle $\gamma$. These three types of solutions are consistent with the local analysis of Vanden-Broeck and Tuck [17]. When surface tension is included in the free surface condition, the angle $\gamma$ can take arbitrary values. It was found that for given values of $\omega, F$ and $\beta$, there is a unique solution. This is in agreement with our everyday experience with jets falling from a nozzle. However, if we interpret the flow as a model for a bubble rising in a tube, this finding is in contradiction with experiments which predict that there is only one bubble for given values of $\omega$ and $\beta$. Couët and Strumolo [3] presented a criterion to select the physically relevant solution which requires $T$ to be different from zero. We have presented an alternative criterion which works in the limit as $T \rightarrow 0$. We have shown that our predicted values of the Froude number and our selected profiles are in as good an agreement with experimental data as those of Couët and Strumolo. 


\section{Acknowledgements}

This work was sponsored by the National Science Foundation.

The first author thanks the GARC and Department of Mathematics, Seoul National University, Korea for their support and the second author thanks the Tel Aviv University, the Technion, Haifa and the Hebrew University of Jerusalem for their hospitality during the time when this paper was put in the final form. Special thanks are also due to Mirna Džamonja for many constructive comments and her help with the Figures.

\section{References}

[1] G. Birkhoff and D. Carter, "Rising plane bubbles", J. Math. and Mech. 6 (1957) 769-779.

[2] R. Collins, "A simple model of a plane gas bubble in a finite liquid", J. Fluid Mech. 22 (1965) 763-771.

[3] B. Couët and G. S. Strumolo, "The effects of surface tension and tube inclination on a twodimensional rising bublle", J. Fluid Mech. 184 (1987) 1-14.

[4] F. Dias and J.-M. Vanden-Broeck, "Flows emerging from a nozzle and falling under gravity", $J$. Fluid Mech. 213 (1990) 465-477.

[5] P. R. Garabedian, "On steady-state bubble generated by taylor instability", Proc. R. Soc. London Ser. A 241 (1957) 423-43.

[6] J. B. Keller, "Teapot effect", J. Appl. Phys. 28 (1957) 859-864.

[7] J. B. Keller and J. Gee, "Flows of thin streams with free boundaries", J. Fluid Mech. 59 (1973) $417-432$.

[8] J. Lee and J.-M. Vanden-Broeck, "Two-dimensional jets falling from funnels and nozzles", Phys. Fluids A 5 (1993) 2454-2460.

[9] C. C. Maneri, "The motion of plane bubbles in inclined ducts", Ph. D. Thesis, Polytechnic Institute of Brooklyn, New York.

[10] M. Reiner, "The teapot effect...a problem", Phys. Today 9 (1956) 16-20.

[11] E.O. Tuck, "Efflux from a slit in a vertical wal", J. Fluid Mech. 1987 (1987) 253-264.

[12] J.-M. Vanden-Broeck, "Bubbles rising in a tube and jets falling from a nozzle", Phys. Fluids 27 (1.984) 1090-1093.

[13] J.-M. Vanden-Broeck, "Rising bubbles in a tube with surface tension", Phys. Fluids 27 (1984) 2604-2607.

[14] J.-M. Vanden-Broeck, "Pointed bubbles rising in a two-dimensional tube", Phys. Fluids 29 (1986) 1343-1344.

[15] J.-M. Vanden-Broeck and J. B. Keller, "Pouring flows", Phys. Fluids 29 (1986) 3958-3961.

[16] J.-M. Vanden-Broeck and J. B. Keller, "Pouring flows with separation", Phys. Fluids A 1 (1989) 156-158.

[17] J.-M. Vanden-Broeck and E. O. Tuck, "Flow near the intersection of a free surface with a vertical wall", SIAM J. Appl. Math. 54 (1954) 1-13. 